\title{
Manejo dietético e suplementar na fisiopatologia da síndrome dos ovários policísticos
}

\author{
Dietary and supplementary management in the pathophysiology of polycystic ovary syndrome \\ Manejo dietético y complementario en la fisiopatología del síndrome de ovario poliquístico
}

Recebido: 08/11/2021 | Revisado: 15/11/2021 | Aceito: 16/11/2021 | Publicado: 25/11/2021

\author{
Elen Chaves de Sousa Xavier \\ ORCID: https://orcid.org/0000-0002-0551-7979 \\ Centro Universitário Fametro, Brasil \\ E-mail: Elen.csx@gmail.com \\ Francisca Marta Nascimento de Oliveira Freitas \\ ORCID: https://orcid.org/0000-0002-0044-0925 \\ Centro Universitário Fametro, Brasil \\ E-mail: Francisca.freitas@fametro.edu.br
}

\begin{abstract}
Resumo
Introdução: A Síndrome do Ovário Policístico (SOP) é um distúrbio endócrino ginecológico que interfere no processo de ovulação e fertilidade feminina. Mulheres com SOP apresentam maiores concentrações de andrógenos circulantes relacionados com Resistência à Insulina (RI), dislipidemia, obesidade, diabetes gestacional, Diabetes Mellitus tipo 2 (DM II), Doenças Cardiovasculares (DCV) e Hipertensão Arterial (HAS) e infertilidade. Objetivo: O objetivo do presente estudo foi o de abordar os efeitos benéficos da terapia nutricional e suplementar na SOP e descrever a patogênese, manifestações clínicas e diagnóstico. Materiais e Métodos: Trata-se de uma revisão de literatura não sistemática de artigos relacionados ao tema da SOP da base de dados SciELO e Pubmed. Resultados e Discussão: A intervenção no estilo de vida da paciente com SOP é a forma de tratamento mais eficiente, sobretudo em mulheres acima do peso, devendo haver manejo dietético e inclusão de atividade física. E nesse contexto, a suplementação nutricional pode ser benéfica. Conclusão: Planos alimentares com controle glicêmico e carga glicêmica controlam à Resistência à Insulina (RI), regredindo o quadro da SOP. Além disso, a suplementação nutricional de mioinusitol e de vitamina D podem contribuir para o controle dos sinais e sintomas da SOP.
\end{abstract}

Palavras-chave: Síndrome dos ovários policísticos; Resistência à insulina; Dietoterapia; SOP Suplementação; SOP; Fisiopatologia da SOP.

\begin{abstract}
Introduction: Polycystic Ovary Syndrome (PCOS) is a gynecological endocrine disorder that interferes with the process of ovulation and female fertility. Women with PCOS have the highest circulating androgen requirements related to Insulin Resistance (IR), dyslipidemia, obesity, gestational diabetes, Type 2 Diabetes Mellitus (DM II), Cardiovascular Disease (CVD) and Arterial Hypertension (SAH) and infertility. Objective: The aim of the present study was to address the beneficial effects of nutritional and supplemental therapy on PCOS and describe its pathogenesis, clinical manifestations and diagnosis. Materials and Methods: This is a non-systematic literature review of articles related to the topic of PCOS from the SciELO and Pubmed databases. Results and Discussion: The intervention in the lifestyle of the patient with PCOS is the most efficient form of treatment, especially in overweight women, with dietary management and inclusion of physical activity. And in this context, nutritional supplementation can be beneficial. Conclusion: Food plans with glycemic control and glycemic load control Insulin Resistance (IR), regressing the PCOS picture. In addition, nutritional supplementation of myoinusitol and vitamin D may contribute to the control of PCOS signs and symptoms.
\end{abstract}

Keywords: Polycystic ovary syndrome; Insulin resistance; Diet therapy; PCOS Supplementation; PCOS; Pathophysiology of PCOS.

\section{Resumen}

Introducción: El Síndrome de Ovario Poliquístico (SOP) es un trastorno endocrino ginecológico que interfiere en el proceso de ovulación y fertilidad femenina. Las mujeres con SOP tienen concentraciones más altas de andrógenos circulantes relacionados con resistencia a la insulina (RI), dislipidemia, obesidad, diabetes gestacional, diabetes mellitus tipo 2 (DM II), enfermedades cardiovasculares (ECV) e hipertensión arterial (HSA) e infertilidad. Objetivo: El objetivo del presente estudio fue abordar los efectos beneficiosos de la terapia nutricional y complementaria en el SOP y describir su patogenia, manifestaciones clínicas y diagnóstico. Materiales y métodos: Se trata de una revisión bibliográfica no sistemática de artículos relacionados con el tema del SOP de la base de datos SciELO y Pubmed. Resultados y Discusión: La intervención en el estilo de vida del paciente con SOP es la forma de tratamiento más eficaz, especialmente en mujeres con sobrepeso, con manejo dietético e inclusión de actividad física. Y en este contexto, la suplementación nutricional puede resultar beneficiosa. Conclusión: Planes alimentarios con control 
glucémico y control de carga glucémica Resistencia a la insulina (RI), retrocediendo el cuadro del SOP. Además, la suplementación nutricional de mioinusitol y vitamina D puede contribuir al control de los signos y síntomas del síndrome de ovario poliquístico.

Palabras clave: Sindrome de ovario poliquistico; Resistencia a la insulina; Terapia dietética; Suplementación de SOP; SOP; Fisiopatología del SOP.

\section{Introdução}

A Síndrome do Ovário Policístico (SOP) é um distúrbio endócrino ginecológico que interfere no processo de ovulação e fertilidade feminina. Ainda com etiologia desconhecida, ela pode afetar 8,7 a 17\% das mulheres em idade reprodutiva de 17 a 39 anos (Zueff, et al., 2012). Os fenótipos da SOP variam, mas existem características comuns, que são os ovários policísticos, irregularidade menstrual, hiperandrogenismo e anormalidades gonadotróficas (Connolly et al., 2015). Mulheres com SOP apresentam maiores concentrações de andrógenos circulantes relacionados com Resistência à Insulina (RI), dislipidemia, obesidade, diabetes gestacional, Diabetes Mellitus tipo 2 (DM II), Doenças Cardiovasculares (DCV) e Hipertensão Arterial (HAS) e infertilidade. Além disso, a SOP é uma das causas da hiperplasia endometrial, sendo então ligada ao câncer endometrial (Costa et al., 2010).

Os sinais e sintomas da SOP incluem hirsutismo, acne, obesidade, dificuldade em perder peso, oligo ou anovulação (os ovários não liberam um óvulo durante um ciclo menstrual regular de 28 dias), oligomenorreia ou amenorreia (ausência de menstruação pelo período de 3 a 6 meses) (Azziz 2016). Para o diagnóstico da SOP é comumente utilizado o método de Rotterdam, em que para uma mulher ser caracterizada com a síndrome é preciso apresentar pelo menos dois de seus três critérios de análise, sendo eles: sinais clínicos ou bioquímicos de hiperandrogenismo, oligo ou anovulação e presença de ovários policísticos em exame de imagem (Legro et al., 2013).

Devido ao desequilíbrio hormonal estabelecido na SOP, os tratamentos convencionais consistem no uso de anticoncepcionais, progestogênios e antiandrogênicos (como metformina e espironolactona). O uso desses medicamentos age contra a formação dos cistos nos ovários, reduz os hormônios androgênicos e controlam a insulina. A terapia medicamentosa é benéfica para o alívio de sinais e sintomas da SOP e para a proteção de possíveis complicações, mas ela pode provocar efeitos colaterais indesejados. Visando a melhor qualidade de vida das mulheres com SOP, é válido destacar que as causas, sinais e sintomas podem ser controlados com o manejo dietético e suplementar, de acordo com o grau de sintomas e complicações da paciente (Rocha, 2011).

Além disso, é importante lembrar que Mulheres com SOP devem manter a alimentação adequada, controle de peso e resistência a inulina, apresentam ovulação normal, podem ter filhos saudáveis. Sendo assim, uma dieta com baixo teor de carboidratos podem reduzir as concentrações a insulina. Estas dietas contribuem para a redução mais efetiva de peso, somando ao maior poder da saciedade das proteínas, se comparadas a carboidratos e lipídios (Santos et al., 2019)

De acordo com Santos et al., (2019) O consumo de alimentos com elevado teor de ácidos graxos poli-insaturados é recomendado para pacientes com SOP, pois, eles melhoram a ação da insulina em tecidos periféricos e diminuem a secreção de insulina pelo pâncreas.

Nesse propósito, o estudo da influência do estilo de vida e hábitos alimentares na SOP são extremamente importantes para a saúde reprodutiva e para o bem-estar geral da mulher. Portanto, o objetivo do presente estudo foi realizar uma revisão de literatura não sistemática de artigos relacionadas aos ao tema de ovários policísticos, incluindo patogênese, manifestações clínicas, diagnóstico e terapia nutricional e suplementar. 


\section{Metodologia}

O estudo foi realizado por meio de uma revisão de literatura não sistemática de caráter descritivo para a compreensão da fisiopatologia, manejo dietético e suplementar da SOP. O procedimento do estudo constituiu em uma pesquisa bibliográfica de materiais em língua portuguesa e estrangeira, constituído por livros, artigos, periódicos e monografias.

Sobre a pesquisa bibliográfica, Lima e Mioto (2007) discorrem:

"A pesquisa bibliográfica implica em um conjunto ordenado de procedimentos que busca por soluções, atento ao objeto de estudo, e que, por isso, não pode ser aleatório.”

Portanto, esse método de pesquisa serve de embasamento para todos os temas pesquisados, comparando opiniões de diferentes autores sobre o mesmo assunto (Lima \& Mioto, 2007).

A coleta de dados foi realizada de forma eletrônica nos bancos de dados de publicações da SciELO e Pubmed. A pesquisa foi direcionada pelas palavras chaves do tema, como: Síndrome dos Ovários Policísticos; Resistência à Insulina; Dietoterapia e SOP; Suplementação e SOP; Fisiopatologia da SOP. O material teórico coletado foi avaliado criteriosamente para descrever bem a temática.

Para a análise de dados foi utilizada a pesquisa qualitativa a fim de argumentar os resultados do estudo por meio de análises e percepções de dados não-mensuráveis. De acordo com Deslauriers (1991), na pesquisa qualitativa, o aluno é sujeito e objeto da pesquisa simultaneamente, pois o conhecimento deve ser imparcial e limitado, já que o desenvolvimento da pesquisa é algo imprevisível.

Na análise de dados há o processo de formação de significados, no qual as informações serão voltadas para descrever os objetivos da temática, destacando a fisiopatologia e os efeitos da dietoterapia e da suplementação alimentar na Síndrome dos Ovários Policísticos.

Na seleção inicial foram selecionados artigos entre 2010 a 2020, 35 publicações; no SciELO foram encontrados 20; Pubmed, 11; Medline, 2, e teses, 4. Assim sendo, 40 publicações compõem o presente estudo, resultantes de pesquisas primárias quantitativas, qualitativas e estudos teóricos. Os artigos encontrados resumem-se em língua portuguesa, inglesa e espanhola. 
Figura 1. Fluxograma detalhando cada etapa do processo de seleção de artigos para a revisão.

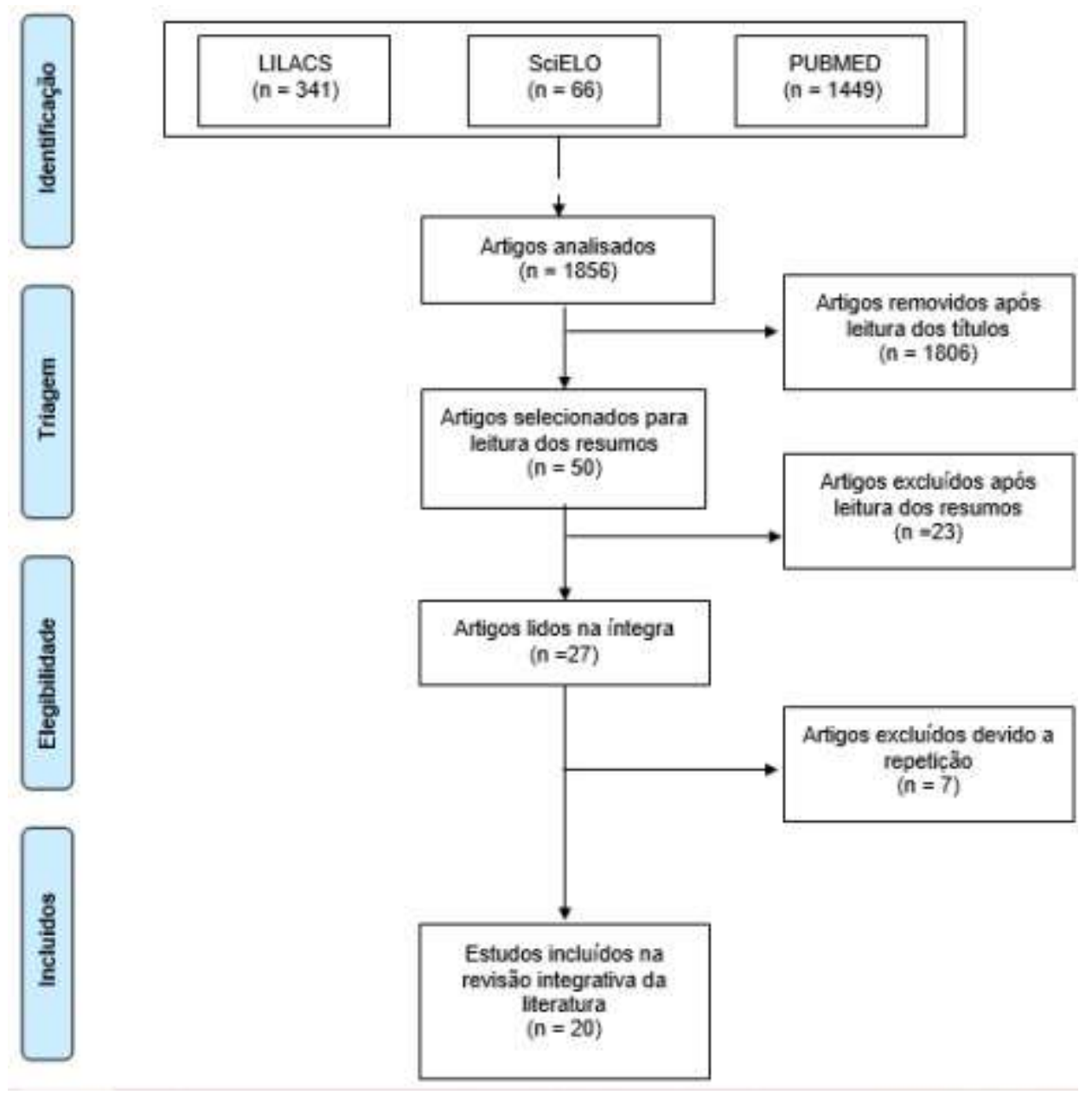

Fonte: Cavalcante et al. (2021).

\section{Resultados e Discussão}

\subsection{Etiologia e fisiopatologia da síndrome dos ovários policísticos}

A Síndrome dos Ovários Policísticos (SOP) é uma doença crônica que foi descrita pela primeira vez em 1935 por Stein e Leventhal, mas sua etiologia ainda é discutida. Ela é caracterizada como um distúrbio metabólico e reprodutor com prevalência de 8,7 a 17,8 \% das mulheres em idade reprodutiva (Zueff et al., 2012). As primeiras manifestações clínicas costumam aparecer no período pós-púbere através da alguns mecanismos fisiopatológicos, como alteração da liberação do hormônio gonadotrofina, defeito na síntese de andrógenos e resistência à insulina (Abbott, 2005).

Uma das teorias propostas para explicar a patogênese da SOP é a perturbação do eixo hipotálamo-hipófise, que resulta na secreção desordenada de gonadotrofina pelo hipotálamo com consequente elevação do hormônio luteinizante (LH) e estimulação baixa ou normal do hormônio folículo estimulante (FSH). Nesse aspecto, a insulina também é um elemento importante na para o desenvolvimento da SOP, pois ela atua em sinergia com o LH para aumentar a produção de andrógenos nas células ovarianas da Teca. A biologia ovariana é o ponto de partida para compreender os antecedentes do desenvolvimento as SOP e as associações patológicas (Duncan, 2014).

\subsubsection{Produção de hormônios nos ovários}

O controle hormonal se dá pelo eixo hipotálamo-hipófise, onde o hipotálamo estimula à hipófise na produção de FSH e LH, os quais atuam nas células ovarianas. Os folículos ovarianos são unidades produtoras de hormônios nos ovários 
compostos por duas camadas de células esteroidogêneses: a Teca e a Granulosa (Minegishi et al., 1989). As células da Teca participam da produção do desenvolvimento do folículo ovariano, da ovulação e da produção de andrógenos; e as células da granulosa produzem estradiol através dos andrógenos da Teca. O LH age na célula da Teca produzindo testosterona a partir do colesterol e o FSH age na célula granulosa, promovendo a conversão da testosterona em estradiol pela enzima aromatase (Tonetta \& Dizerega, 1989).

$\mathrm{Na}$ mulher com SOP existe um aumento da síntese de LH, produzindo muita testosterona e redução de FSH, reduzindo à conversão dessa testosterona em estradiol. Dessa forma as concentrações de testosterona ficam mais elevadas na corrente sanguínea e mais bi disponíveis. O aumento da exposição dos ovários aos andrógenos interfere no crescimento e desenvolvimento dos folículos ovarianos, estimulando o crescimento de folículos antrais menores e inibindo o crescimento folicular posterior, ou seja, reduz o desenvolvimento e morte dos folículos (Child et al., 2001). A resposta dos ovários à exposição aumentada de andrógenos é o desenvolvimento de morfologia policística, com pequenos folículos antrais cheios de fluidos, claramente visíveis em exame de ultrassom, resultando em um aumento da incidência de anovulação. Os folículos antrais com fluidos podem crescer normalmente com administração de FSH exógeno (Balen, 2013).

\subsubsection{Regulador da biodisponibilidade androgênica}

Um fator importante a ser considerado é a existência de regulador da biodisponibilidade androgênica denominado de Globulina Ligadora de Hormônios Sexuais (SHBG), que elimina o excesso de andrógenos para evitar interferência no crescimento e desenvolvimento do folículo ovariano (Franks, 2012). Para essa neutralização androgênica ocorrer, os níveis de SHBG precisam estar elevados, fator que geralmente não é observado na SOP, sobretudo se a mulher estiver obesa. É válido destacar que a proteína SHGB é inversamente proporcional ao peso corporal, ou seja, quanto mais elevado for o peso do indivíduo, menor os níveis de SHBG, por isso uma das formas de controlar a SOP é gerenciar o peso corporal. Na mulher com SOP é comum observar níveis elevados de LH e níveis baixos de FSH e SHBG, aumentando ainda mais a biodisponibilidade da testosterona, piorando a SOP. À medida que o peso diminui, o SHBG aumenta, reduzindo a biodisponibilidade de andrógenos, melhorando a SOP. Portanto, indiretamente, o peso é um controlador da ação do LH nos ovários (Motta, 2012).

\subsubsection{A insulina e a síntese de andrógenos nos ovários}

A insulina é um hormônio que regula o transporte de glicose e tem ação anabólica (DUNAIF, 1997). Além disso, ela é cofator da regulação da síntese de andrógenos dependentes de LH nas células da Teca, sendo assim, o LH tem efeito multiplicador de androgênios através da insulina. Na resistência à insulina (RI), existe uma dificuldade da insulina se ligar ao receptor, prejudicando a cascata de fosforilação que desloca do GLUT 4 da membrana para levar glicose dentro para dentro da célula. Consequentemente, a tolerância de glicose é reduzida, forçando o organismo a produzir mais insulina para promover a captação de glicose, gerando uma hiperinsulinemia. Alguns tecidos são muito resistentes à insulina, como o tecido adiposo e o tecido muscular, mas os ovários permanecem sensíveis à insulina. Sendo assim, na hiperinsulinemia associada à RI, os ovários são muito mais estimulados, produzindo mais andrógenos nas células da Teca, piorando a SOP (Zhang, Garmey \& Veldhuis, 2000).

A insulina é um hormônio anabólico, isso significa que altos níveis de insulina estão associados à facilidade de ganho de peso corporal e dificuldade em perda de peso. À medida que o peso aumenta, a RI e hiperinsulinemia também aumentam, gerando um ciclo vicioso entre peso corporal e RI. Portanto, existe essencialmente três fatores motivadores para aumentar a produção de andrógenos nos ovários: aumento de LH, hiperinsulinemia por RI e aumento de peso (Heilbronn et al., 2013). De acordo com BARBER (2015), 50 a 90\% das pacientes com SOP manifestam RI e nesse ponto, o manejo dietético é essencial para controlar a RI e reduzir a produção de androgênios ovarianos (Nahum, Thong \& Hillier, 1995). 


\subsubsection{Papel da resistência à insulina na fisiopatologia da SOP}

A Hipersinulinemia por RI modifica o metabolismo do colesterol, levando à uma dislipidemia; reduz os níveis de SHBG no fígado, aumentando a testosterona livre; reduz o transporte de glicose, provocando disglicemia; aumenta a liberação de LH pela hipófise, estimulando a síntese de androgênicos ovarianos; e aumenta as enzimas chaves na conversão de testosterona, como a dehidroepiandrosterona (DHEA) na adrenal, aumentando a biodisponibilidade de andrógenos. Juntos, esses fatores provocados pela RI pioram consideravelmente as características androgênicas e metabólicas da SOP, bem como as manifestações clínicas. A RI pode ser contornada por meio da perda de peso, terapia medicamentosa e manejo dietético (Barber et al., 2015).

\subsection{Manifestações clínicas da SOP: acne e hirsutismo}

Os cravos e acnes como manifestações clínicas da SOP estão correlacionados com a enzima $5 \alpha$-redutase, que converte a testosterona em diidrotestosterona na unidade pilo-sebácea, estimulando as glândulas sebáceas na produção de sebo e promovendo uma hiperqueratinização folicular (comedogênese). Esses fatores favorecem a proliferação do Propionibacterium acnes e o processo de inflamação dérmica, contribuindo para o aparecimento de cravos e acne (Yildiz, 2008).

O hisurtismo é o crescimento excessivo de pelos terminais em mulheres em um padrão masculino, afetando 5 a $10 \%$ das mulheres de forma geral, sendo que $80 \%$ delas apresentam a SOP. O diagnóstico clínico do hirsutismo é o hiperandrogenismo, pois a alta produção de andrógenos, aumenta a sensibilidade dos folículos pilosos aos hormônios, transformando os pelos vêllus (finos, curtos e pouco pigmentados) em pelos terminais (grossos, compridos e mais pigmentados) de forma irreversível (Yildiz, 2008).

\subsection{Manejo dietético na SOP}

Como citado anteriormente, a insulina tem papel importante na SOP, por isso o principal fator do manejo dietético é o controle glicêmico a fim de reduzir a RI, melhorando a sensibilidade à insulina (Mckittrick, 2002). O alto consumo de carboidratos refinados leva ao aumento do índice glicêmico e inflamação crônica de baixo grau. Isso provoca o aumento da RI, aumento de citocinas inflamatórias e dificulta a produção de SHBG pelo fígado. Com o controle do consumo de carboidratos na dieta é possível controlar a glicemia, melhorar o perfil lipídico, regular os ciclos menstruais, auxiliar a perda de tecido adiposo e melhorar os marcadores inflamatórios. Desse modo, o manejo dietético consiste em uma dieta com baixo índice glicêmico (IG), com baixa carga glicêmica (CG) e com característica anti-inflamatória (Foster et al., 2003).

Dentre as estratégias para reduzir o IG e a CG da dieta está a inclusão das fibras na alimentação, sobretudo a fibra solúvel, pois ela tem um maior impacto na resposta glicêmica (Gambineri et al, 2002). As fibras solúveis aumentam a viscosidade do bolo alimentar e reduzem a atividade de enzimas digestivas, impactando na digestão e absorção de nutrientes. Dessa forma, há uma regulação da glicemia pós pandrial e consequente melhora da resposta insulínica e regulação do apetite, além de reduzir os níveis de colesterol. Pectina, mucilagens e beta-glucana são fibras solúveis que podem ser incluídas na dieta pelo consumo de farinha da casca de berinjela e de maracujá, casca da maçã, goma guar, psyllium, farelo de aveia e cevada. O amido resistente tem um papel fisiológico parecido com as fibras, pois ele aumenta a secreção de ácidos biliares, reduzindo as concentrações séricas de triglicerídeos e colesterol, além de controlar a glicemia. O amido resistente está presente nos grãos, farinhas e leguminosas (Mello \& Laaksonen, 2009).

Outros alimentos podem auxiliar na modulação da resposta glicêmica como, o vinagre de maçã, canela, semente de chia, biomassa de banana verde e gorduras mono e poliinsaturadas (presente no abacate, nozes, azeite de oliva, sementes) (Mello \& Laaksonen, 2009). 


\subsection{Mioinusitol}

O inusitol é um composto químico da classe do álcool amplamente encontrado na natureza com nove isoformas, onde o mioinusitol é mais comum, estando presente em tecidos animais e vegetais (Clements \& Darnell, 2008). No passado, o mioinusitol foi definido como "açúcar miometral", pois ele pode ser produzido por células humanas a partir da glicose (Reynolds, 1993). Alguns estudos mostraram que a molécula de inusitol está diretamente envolvida na sinalização celular de insulina, onde a sua deficiência do contribui para a RI (Baillargeon et al, 2008).

A administração de mioinusitol em mulheres com SOP mostrou redução da RI, como no estudo de metanálise realizado por ZENG e YANG em que foi ofertado de $500 \mathrm{mg}$ a $2 \mathrm{~g}$ de mioinusitol para mulheres com SOP. Ao comparar o grupo controle com o grupo teste foi observado que as mulheres que suplementaram com mioinusitol tiveram uma melhora da RI e um efeito no eixo hipotálamo-pituitário-ovariano, reduzindo o LH e aumentando SHBG e estradiol. Portanto, a suplementação de mioinusitol para pacientes com SOP e RI pode ser benéfico (Zeng \& Yang, 2017).

\subsection{Vitamina D}

A vitamina $\mathrm{D}$ é um dos fatores nutricionais associados à SOP, principalmente em mulheres obesas, que apresentam prevalência de baixa dessa vitamina (Thomson, Spedding \& Buckley, 2012). Uma das explicações baseia-se no fato da vitamina D ser lipossolúvel, sendo então acumulada no tecido adiposo, reduzindo sua biodisponibilidade (Lagunova et al., 2009). Alguns estudos têm indicado concentrações insuficientes de $25(\mathrm{OH}) \mathrm{D}$ circulante relacionado ao hiperandrogenismo, síndrome metabólica, RI, aumento de massa corporal e de percentual de gordura (WEHR et al., 2009). Além disso, outro fator percebido na etiologia das pacientes com SOP são distúrbios na atividade dos receptores de vitamina D (VDR) expressos em vários tecidos, incluindo os ovários (Ding, Gao, Wilding, Trayhurn \& Bing, 2012).

Um estudo realizado por JAFARI e colaboradores dividiu um grupo de mulheres em três: o primeiro grupo recebeu 4000 UI de vitamina D, o segundo grupo recebeu 1000 UI de vitamina D e o terceiro grupo recebeu placebo. Eles perceberam que o grupo de mulheres que recebeu 4000 UI de vitamina D obteve redução da glicose, redução sérica de insulina e melhora da RI e da relação colesterol/HDL. Portanto, a suplementação de vitamina $\mathrm{D}$, principalmente associada à uma dieta para perda de peso tem efeitos benéficos ao hiperandrogenismo nas mulheres com SOP (Jafari et al., 2017).

\section{Considerações Finais}

A Síndrome dos Ovários Policísticos é um distúrbio crônico, endócrino e ginecológico que está intimamente relacionada à RI, sobrepeso e obesidade. O eixo hipotálamo-hipofisário e a produção de hormônios ovarianos são o ponto de partida para compreender sua fisiopatologia. De forma geral, na mulher com SOP percebe-se um aumento do hormônio LH e redução de FSH e SHBG, que implica em uma menor conversão de testosterona em estradiol, deixando esse andrógeno mais biodisponível.

A acne, o hirsutismo e morfologia policística nos ovários estão inclusos na sintomatologia da SOP como consequências direta do aumento de andrógenos na corrente sanguínea. E nesse contexto, a hiperinsulinemia é um fator importante para a piora dos sinais e sintomas, pois os ovários permanecem sensíveis à insulina na RI, produzindo mais andrógenos nas células da TECA.

O controle do peso corporal e a composição dietética são de extrema importância para melhorar a resposta hormonal da mulher com SOP, minimizar a sintomatologia e reduzir os riscos metabólicos associados. No manejo dietético, o controle do consumo de carboidrato está associado à melhora da glicemia, da RI da composição corporal e da inflamação crônica característica da SOP. 
As estratégias alimentares que se encaixam nesse contexto consideram uma alimentação com redução do IG e da CG, bem como a inclusão de fibras, sobretudo solúveis, para impactar na resposta glicêmica do organismo. As dietas mediterrânea, cetogênica e low carb apresentam essas características e ainda tem um perfil anti-inflamatório, as quais podem ser recomendadas de acordo com o nível de RI da paciente.

Do ponto de vista suplementar, mulheres com SOP parecem se beneficiar com a ingestão de mioinusitol e vitamina D como complemento do manejo dietético. Na suplementação de $500 \mathrm{mg}$ a $2 \mathrm{~g}$ de mioinusitol houve um efeito benéfico na RI, redução de LH e aumento de SHBG. E a suplementação de 4000 UI de vitamina D em mulheres com SOP mostrou redução da glicose sérica e de insulina, além da melhora da relação colesterol/HDL. Assim, uma dieta que tenha controle glicêmico associado à ingestão de mioinusitol e vitamina $\mathrm{D}$ podem ser vistos como proposta de intervenção para melhora da sintomatologia e da qualidade de vida da mulher com SOP.

\section{Referências}

Abbott, D. H., Barnett, D. K., Bruns, C. M. et al. (2005). Androgen excesso fetal programming of female reproduction: a developmental aetiology for polycyr polycystic ovary syndrome? Hum Reprod Update. 11, 357-374.

Azzi, R. (20016). PCOS in 2015: New insights into the genetics of polycistics ovary syndrome. Nat Rev Endocrinol, 12(2), 74-5.

Baillargeon, J. P., Nestler, J. E., Ostlund, R. E., Apridonidze, T., \& Diamanti, E. K. (2008). Greek hyperinsulinemic women, with or without polycystic ovary syndrome, display altered inositol metabolism. Hum Reprod, 24, 139-44.

Balen, A.H. (2013). Ovulation induction in the management of anovulatory polycystic ovary syndrome. Mol Cell Endocrinol, 373 , 77-82.

Barber, T.M., et al. (2015). Polycystic ovary syndrome: insight into pathogenesis and a common association with insulin resistance. Clinical Medicine. 15(6), 72-76.

Cavalcante, I. dos S. et al. (2021). Síndrome dos ovários policísticos: aspectos clínicos e impactos na saúde da mulher. Research, Society and Development, 10(2), e23810212398. https://doi.org/10.33448/rsd-v10i2.12398.

Child, T. J., Abdul-Jail, A. K., \& Gulekli, B. et al. (2001). In vitro maturation and fertilization of oocytes from unstimulated normal ovaries, polycystic ovaries, and women with polycystic ovary syndrome. Fertil Steril, 76, 936-942.

Clements, R., \& Darnell, B. (1980). Myo-inositol content of common foods: development of a high-myo-inositol diet. Am J Clin Nutr. 33, $1954-67$.

Connolly, F., Era, M.T., Spath, K., Boswell, L. Mcneilly, A. S., \& Duncan, W.C. (2015). In a Ovine Model of Polycystic Ovary Syndrome (PCOS) prenatal androgens suppress female renal gluconeogenesis. PLos One. 10(7), e0132113

Costa, E. C., Soares, E. M. M., Lemos, T. M., Maranhão, T. M. O., \& Azevedo, G. D. Índices de obesidade central e fatores de risco cardiovascular na síndrome dos ovários policísticos. Arq Bras Cardiol. 2010; 94(5):633-8.

Deslauriers, J. P. (1991). Recherche qualítative - Guide pratique. Montreal: McGraw Hill.

Ding, C., Gao, D., Wilding, J., Trayhurn, P., \& Bing, C. (2012). Vitamin D signalling in adipose tissue. Br J Nutr, $108(11), 1915-192$.

Dunaif, A. (1997). Insulin resistance and the polycystic ovary syndrome: mechanism and implications for pathogenesis. Endocr Ver. 18, 774-800.

Duncan, W. C. (2014). A guide to understanding polycystic ovary syndrome (PCOS). Journal of Family planning and reproductive health care, 217-225.

Foster, G. D., \& Wyatt, H. R. et al. (2003). A randomized trial of a low-carbohydrate diet for obesity. N engl J Med. $2082-90$.

Franks, S. (2012). Polycystic ovary syndrome. N Engl J Med 1995, 333,835-861. 3

Motta, A. B. The role of obesity in the development of polycystic ovary syndrome. Curr Pharm Des, 18, $2482-2491$.

Gambineri, A., Pelusi, C., et al. (2002). Obesity and the polycystic ovary syndrome. Int J Obes Relat Metab Disord, 883-96.

Heibronn, L. K., Coster, A. C., Campbell, L. V. et al. (2013). The effect of short term overfeeding on serum lipids in healthy humans. Obesity, 21, E649E659.

Jafari, S. S., et al. (2017). The efect of vitamin D supplementation in combination with low-calorie diet on anthropometric indices and androgen hormones in women with polycystic ovary syndrome: a double-blind, randomized, placebo-controlled trial. J Endocrinol Invest.

Lagunova, Z., Porojnicu, A. C., Lindberg, F, Hexeberg, S., \& Monan, J. (2009). The dependency of vitamin D status on body mass index, gender, age and season. Anticancer Re, 29(9), 3713-3720.

Lima, T. C. S., \& Mioto, R. C. T. (2007). Procedimentos metodológicos na construção do conhecimento científico: a pesquisa bibliográfica. Katál, Florianópolis, 10. 
Research, Society and Development, v. 10, n. 15, e237101522975, 2021

(CC BY 4.0) | ISSN 2525-3409 | DOI: http://dx.doi.org/10.33448/rsd-v10i15.22975

Legro, R. S. et al. (2013). Diagnostic and treatment of polycystic ovary syndrome: An Endrocrine Society practice guideline. The journal of clinical Endrocrinology \& Metabolism, 4565-4592.

Mello, V. D., \& Laaksonen, D. E. (2009). Fibras na dieta: tendências atuais e benefícios à saúde na síndrome metabólica e no diabetes melito tipo 2. Arq Bras Endocrinol Metab, 53.

Minegishi, T., Tano, M., \& Igarashi, M. et al. (1997)). Expression of follicle-stimulating hormone receptor in human ovary. Eur J Clin Invest, $27,469-474$.

Motta, A. B. (2012). The role of obesity in the development of polycystic ovary syndrome. Curr Pharm Des. 18, $2482-2491$.

Nahum, R., Thong, K. J., \& Hillier, S. G. Metabolic regulation of androgen production by human thecal cells in vitro. Hum Reprod, 10 , 75-81.

Reynolds, J. E. (1993). An isomer of glucose that has traditionally been considered to be a B vitamin although it has an uncertain status as a vitamin and a deficiency syndrome has not been identified in man. Martindale-the Extra Pharmacopoeia, 30th ed. Pennsylvania: Rittenhouse Book Distributors, 1379

Rocha, M. P., Marcondes, J. A., Barcellos, C. R., Hayashida, S. A., Curi, D. D., Fonseca, A. M., da et al. (2011). Dyslipidemia in women with polycystic ovary syndrome: incidence, pattern and predictors. Gynecol Endocrinol. 814-9.

Santos, S. S., et al. (2019). Aspectos nutricionais e manejo alimentar em mulheres com síndrome dos ovários policísticos. Revista saúde em foco. Edição 11.

Thomson, R. L., Spedding, S., \& Buckley, J. D. Vitamin D in the aetiology and management of polycystic ovary syndrome. Clin Endocrinol, 77(3), 343-350, 2012.

Tonetta, S. A., \& Dizerega, G. S. (1989). Intragonadal regulation of follicular maturation. Endocr Ver, 10, $205-229$.

Wehr, E. Pilz, S., Schweighofer, N., Giuliani, A., Kopera, D. Pieber, T., et al. (2009). Association of hypovitaminosis D with metabolic disturbances in polycystic ovary syndrome. Eur J Endocrinol, 161(4), 575-582.

Yildiz, B. O. (2008). Assessment, diagnosis and treatment of a patient with hirsutism. Nature Clinical Pratice Endocrinology \& Metabolism.

Zeng, L., \& Yang, K. (2017). Effectiveness of myoinositol for polycystic ovary syndrome: a systematic review and meta-analysis. Endocrine, 59 (1): $30-38$.

Zhang, G., Garmey, J. C., \& Veldhuis, J. D. (2000). Interactive stimulation by luteinizing hormone and insulin of the steroidogenic acute regulatory (StAR) protein and 17alpha-hydroxylase/17,20-lyase (CYP17) genes in porcine theca cells. Endocrinology, 141, 2735-2742.

Zuef, L. F. N., Marti, W. P., Vieira, C. S., \& Ferriani, R. A. (2012). Ultrasonographic and laboratory markers of metabolic and cardiovascular disease risk in obese women with polycystic ovary syndrome. Ultrasound Obstet Gynecol., 39(3), 341-7. 